\title{
Reactive Power Limits in Distributed Generators from Generic Capability Curves
}

\author{
Gustavo Valverde, Member, IEEE and Juan J. Orozco \\ Electrical Power Engineering Research Laboratory \\ School of Electrical Engineering, University of Costa Rica \\ 11501-2060 UCR, San José, Costa Rica \\ gvalverde@eie.ucr.ac.cr, jorozco@cndcr.com
}

\begin{abstract}
This paper presents a generic trapezoid-type capability curve for any distributed generation technology. This curve is used to estimate the machine reactive power limits in terms of the actual active power and terminal voltage. The proposed simplification was tested on synchronous machines, doubly fed induction generators and full power converter interfaced generators. This methodology can be implemented in Volt/VAR control schemes when the estimation of DG capability is required.

Index Terms-Voltage control, distributed generation, distribution network, reactive power capability, synchronous machine, doubly fed induction machine, full power converters, solar arrays.
\end{abstract}

\section{INTRODUCTION}

Distributed generators (DG) can absorb or inject reactive power for voltage regulation and operation optimization of the grid [1]. Examples of these opportunities are reported in [2], [3] and [4]. Although many control schemes have assumed fixed DG power limits, reactive limits vary depending on the actual generator active power $P$ and terminal voltage $V$ [5].

Fig. 1 presents the typical reactive power capacity of a DG as a function of active power production and terminal voltage. Note that reactive capacity increases as $P$ reduces. If the DG reactive capacity is fixed at $Q_{\text {lim }}$, the generator capacity will be underused for low $P$ values. Hence, updated reactive power limits could be used to take full advantage of DG capabilities.

Actual reactive power limits can be calculated from generator parameters and their respective limiting factors [6]. Unfortunately, these calculations usually require too much information from (most privately owned) DGs, making it difficult to be used by centralized control schemes and on-line applications. Thus, there is a need for estimating the reactive power limits from limited information of DGs.

The estimation should be computationally efficient for online applications while keeping a reliable level of accuracy. A simplistic model could lead to violation of DG actual limits or impose over restrictions on generators power outputs.

This paper proposes a simplified method to calculate reactive power limits of distributed generators based on parameterized generic capability curves. These curves are made of eight points extracted from the actual capability curves for two different terminal voltages. Interpolation is later used to calculate the generator reactive power limits.

The remaining of this paper is organized as follows: The main limiting factors of different DG technology is summa-

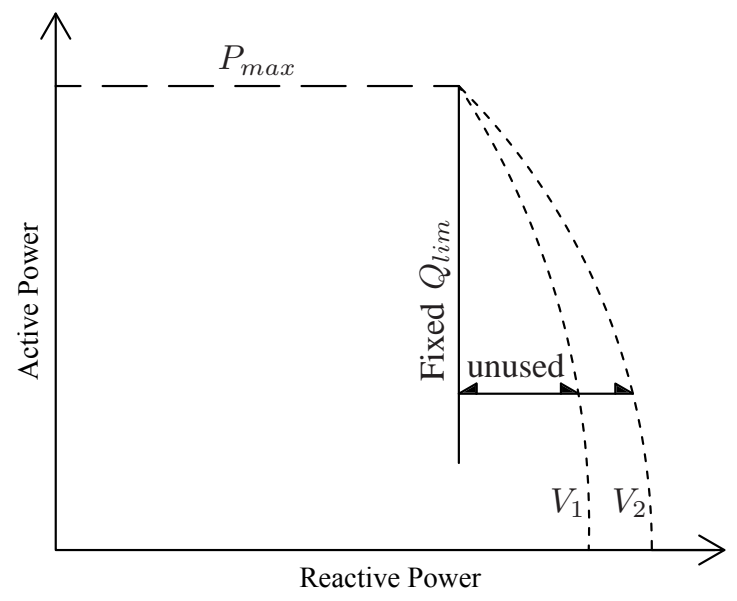

Figure 1. Fixed and actual reactive power limits

rized in Section II. The proposed methodology to estimate DG reactive power limits is introduced in Section III. Results and conclusions are presented in Sections IV and V, respectively.

\section{CApability Curve}

The capability curve defines the machine permissible operating region for a given terminal voltage $V$ [6]. This region is generally bounded by equipment limitations expressed by maximum voltage or current limits. The following sub-sections summarize some of these limits in different DG technologies. The reader should recall that these limits (and approximations in Section III) are often true, but other factors not mentioned here may lead to more or less conservative estimates of the actual capabilities. To be sure, vendors should be asked to provide information regarding DG capability at different operating conditions.

\section{A. Synchronous Machines}

Synchronous generators can be found in distribution networks driven by small hydro and thermal turbines. The main limiting factors are listed below:

- Armature Current Limitation: This limit is defined by the allowable armature winding heating, expressed in terms of a maximum armature current $I_{a}^{\max }$. This limit corresponds to a circle centered at the origin of the Q-P plane with radius $V I_{a}^{\max }$. 


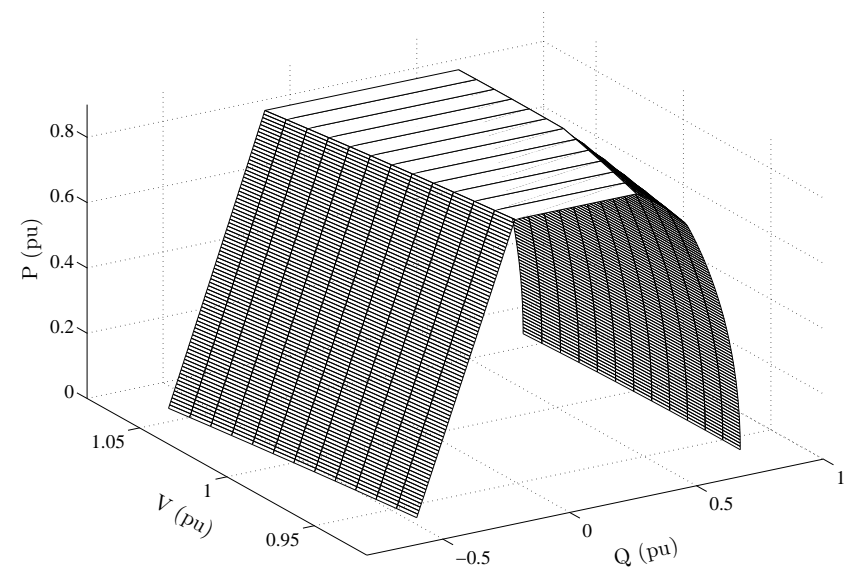

Figure 2. Capability polyhedron of synchronous generator

- Field Current Limitation: This limit is defined by the allowable field winding heating, expressed in terms of a maximum field current $I_{f}^{\max }$. When rotor saliency and core saturation are taken into account, the field capability curve is obtained when solving a set of non-linear equations given the active power $P, V$ and $I_{f}^{\max }$, for details see [6].

As opposed to the armature current limitation, the field winding limit is more restrictive for higher terminal voltage operation. In addition, the limiting factor at lagging power factor is generally the field winding heating. Hence, synchronous machines are typically equipped with an overexcitation limiter (OEL).

- Under-excitation Limitation: At leading power factor the reactive power absorption is limited by the theoretical stability limit (loss of synchronism) and stator core end heating [7]. The latter is generally more restrictive than the stability limit in steam turbine generators [8]. Hydro generators are not limited by end-region heating because of their different construction [9].

- Mechanical Power Limitation: This is the maximum mechanical power that can be extracted from the turbine. Generally, the turbine is sized to match the generator real power at rated power factor.

Fig. 2 shows a family of capability curves for a hydro driven synchronous machine for different terminal voltages [10]. The armature and field current limitation are calculated based on the procedure explained in [6], including saturation and rotor saliency effects. Clearly, the field current limitation (lagging p.f.) becomes more restrictive for higher terminal voltages.

The sub-excitation limit (at leading power factor) obeys a maximum internal angle $\varphi^{\max }$. Note that this limit becomes less restrictive for higher terminal voltages, similar to the armature current limitation (upper cut in over-excitation region).

A comprehensive set of other limiting factors is presented in [8]. These extra factors may include minimum turbine output, plant and system operating conditions.

\section{B. Doubly Fed Induction Generator}

This generator is made of a wound rotor induction machine whose stator winding is directly connected to the grid while the rotor winding is excited through a power electronic converter system. The latter consists of two independently controlled back-to-back converters: the rotor-side converter (RSC) and the grid-side converter (GSC) [11]. The main limiting factors on DFIGs are as follows:

- Stator Current Limitation: This is defined by the allowable stator winding heating expressed in terms of a maximum stator current $I_{s}^{\max }$ [12]. This limit corresponds to a circle centered at the origin of the Q-P plane with radius $V I_{s}^{\max }$.

- Rotor Current Limitation: It defines the stator reactive power limit such that the rotor current does not exceed the maximum value $I_{r}^{\max }$. At lagging power factor the rotor current limit is more restrictive than the stator limit. On the contrary, the stator heating imposes more restrictions for leading power factor operation [12], [13]. However, due to its inductive nature, the DFIG can absorb large amounts of reactive power through the stator [14].

- Rotor Voltage Limitation: It corresponds to the machine reactive power limitation due to maximum rotor voltage. This limit is of interest for low active power production, when the rotor approaches low speeds. However, as the machine is disconnected at low wind speeds, the maximum rotor voltage may not be met in practice.

- GSC Limitation: The GSC is limited due to overloading constraints. In general, the converter is designed to export about $25 \%$ of the machine active power output at full load. Although the converter can exchange reactive power, it is limited depending on the active power imported or exported by the rotor [11]. The latter is approximately:

$$
P_{r}=-s P_{s}
$$

where $s$ is the rotor slip and $P_{s}$ is the active power output in the stator. Clearly, the maximum reactive power exchange in the GSC occurs at synchronous speed $(s=0)$. Note that at high or low rotor speeds the reactive power capability of the GSC is more restricted.

- Wind Turbine Limitation: This is the maximum mechanical power that can be extracted from the turbine blades. At high (cut-off) wind speeds the machine will be disconnected from operation.

The capability polyhedron in Fig. 3 represents a family of capability curves for a DFIG at different terminal voltages. These curves were obtained following the formulation in [11].

As presented in Fig. 3, the stator (at leading power factor) and the rotor current limitation (at lagging power factor) become less restrictive for higher terminal voltage operation [14]. However, the rotor voltage limitation represented by the lower plane becomes more restrictive for higher terminal voltage operations. Note that at low active power and $V>1.02 \mathrm{pu}$ the machine cannot produce reactive power i.e. $Q<0$.

Operating limits other than those presented in Fig. 3 may be considered as well. They may include saturation, machine losses and stator Y-connection, for details see [11] and [12]. 


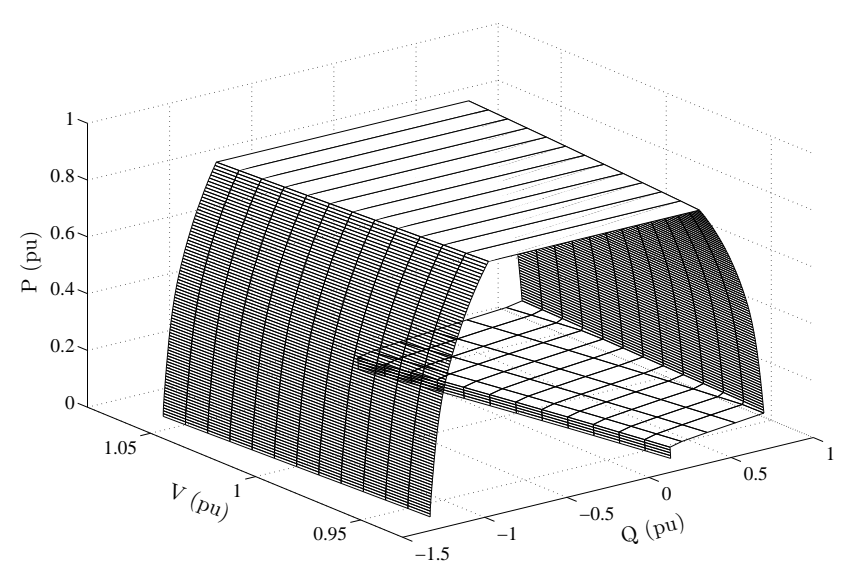

Figure 3. Capability polyhedron of doubly fed induction generator

\section{Full Converter Interfaced Generators}

Photovoltaic (PV) systems and Type-4 wind generators are connected to the grid by means of full power converters. In this paper, the reactive power capability will be focused on the converter limitations rather than generating unit restrictions.

In general the power electronic interface consists of two back-to-back power converters. The following are the main limiting factors of full converter interfaced generators (FCG):

- Converter Current Limitation: This corresponds to the maximum permitted heating of the power converter expressed in terms of a maximum converter current $I_{c}^{\max }$ [15]. Similar to the armature current limitation of a synchronous machine, this limit corresponds to a circle centered at the origin of the Q-P plane with radius $V I_{c}^{\max }$.

- Converter Voltage Limitation: It is determined by the maximum DC-link voltage in the power converter, expressed in terms of a maximum voltage $V_{c}^{\max }$ [15]. This is similar to the field current limit of a synchronous generator [16].

At lagging power factor the converter voltage limit is more restrictive than the current limitation. On the contrary, the converter current imposes more restrictions for leading power factor operation.

- Active Power Limitation: This is the maximum active power that can be extracted from the generating unit (solar arrays, permanent magnet, synchronous or induction machine).

Fig. 4 presents a family of FCG capability curves for different terminal voltages. These curves were obtained from the formulation presented in [15] and [17].

The polyhedron in Fig. 4 shows that for higher terminal voltages, the FCG can produce less $Q$ for a given $P$ value due to the $V_{c}^{\max }$ restriction. This is the opposite effect for the current limitation at leading power factor. Here, the FCG can absorb more reactive power $(Q<0)$ at higher terminal voltages.

\section{Generic CAPability Curves}

As presented before, the capability curves depend on machine parameters, thermal limits and terminal voltage. This

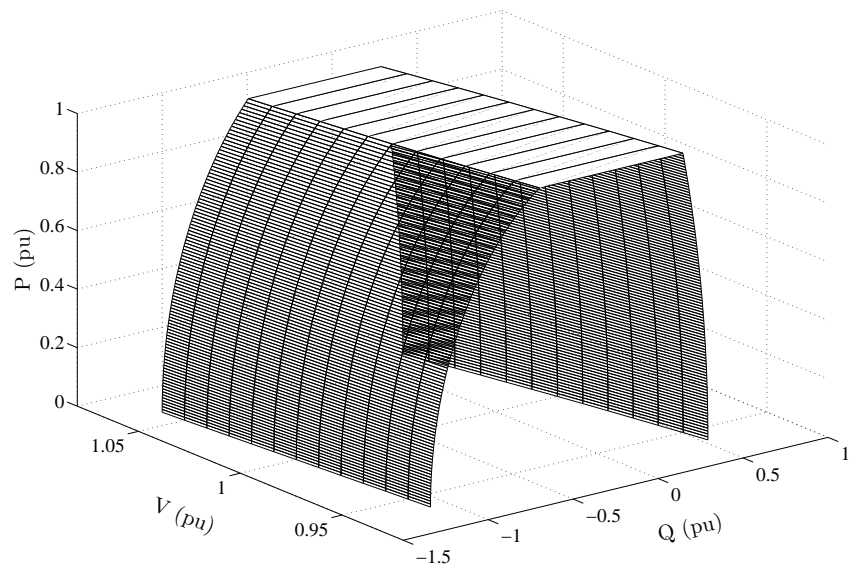

Figure 4. Capability polyhedron of full converter interfaced generator

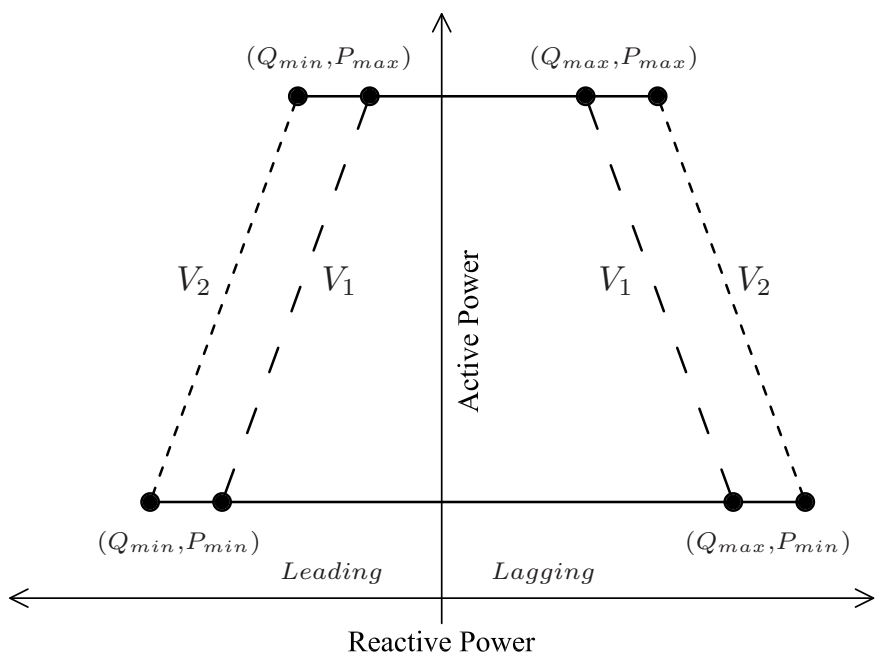

Figure 5. Trapezoid-type generic capability curves

section presents generic curves made of eight points extracted from the actual capability curves for two different terminal voltages.

Fig. 5 presents the proposed generic capability curves. The curves use the minimum and maximum machine active power limits to define the bases of the trapezoids. The legs are defined by the minimum and maximum reactive powers at both $P_{\min }$ and $P_{\max }$, for two different terminal voltages $V_{1}$ and $V_{2}$.

Take for instance the capability curve of a synchronous machine for $V_{1}=0.96 \mathrm{pu}$ and $V_{2}=1.04 \mathrm{pu}$, as shown in Fig. 6. Four points are extracted for each voltage value: $\left(Q_{\min }, P_{\min }\right),\left(Q_{\max }, P_{\min }\right),\left(Q_{\min }, P_{\max }\right)$ and $\left(Q_{\max }, P_{\max }\right)$. Given the actual active power production and machine terminal voltage, it is possible to determine the reactive power limits of the machine based on interpolation between the trapezoids. For a given $P$ value, the maximum and minimum reactive powers are computed for $V_{i}$, with $i=1,2$ :

$$
Q_{\max }^{V_{i}}=Q_{\max }^{P_{\max }}+\left(Q_{\max }^{P_{\max }}-Q_{\max }^{P_{\min }}\right)\left(\frac{P-P_{\max }}{P_{\max }-P_{\min }}\right)
$$




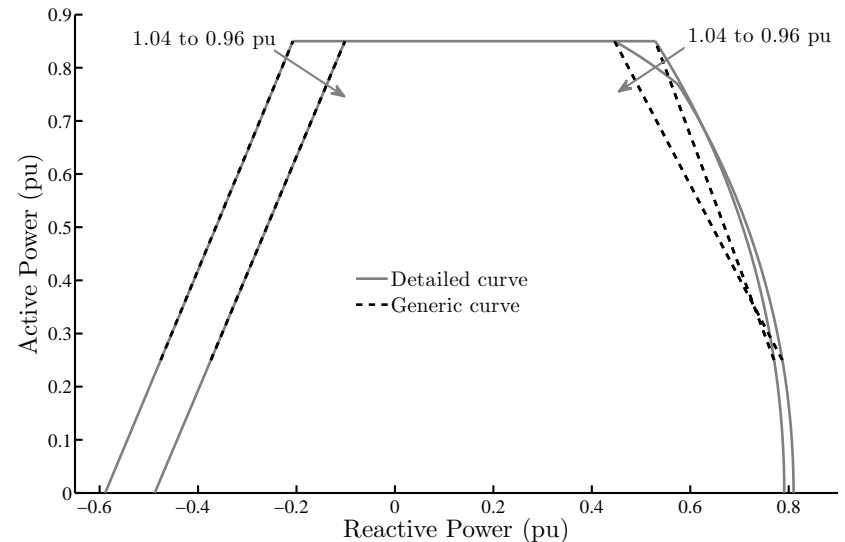

Figure 6. Synchronous machine capability curves

$$
Q_{\min }^{V_{i}}=Q_{\min }^{P_{\max }}+\left(Q_{\min }^{P_{\max }}-Q_{\min }^{P_{\min }}\right)\left(\frac{P-P_{\max }}{P_{\max }-P_{\min }}\right)
$$

where $Q_{\max }^{P_{\max }}$ stands for $Q_{\max }$ at $P_{\max }$ for a given $V_{i}$ voltage. The reader can follow the meaning for $Q_{\max }^{P_{\min }}, Q_{\min }^{P_{\max }}$ and $Q_{\min }^{P_{\min }}$. Finally, the reactive power limits $Q_{\max }$ and $Q_{\min }$ are estimated for the actual machine terminal voltage $V$ :

$$
\begin{gathered}
Q_{\max }=Q_{\text {max }}^{V_{1}}+\left(Q_{\text {max }}^{V_{1}}-Q_{\text {max }}^{V_{2}}\right)\left(\frac{V-V_{1}}{V_{1}-V_{2}}\right) \\
Q_{\min }=Q_{\text {min }}^{V_{1}}+\left(Q_{\text {min }}^{V_{1}}-Q_{\text {min }}^{V_{2}}\right)\left(\frac{V-V_{1}}{V_{1}-V_{2}}\right)
\end{gathered}
$$

where $V_{1} \neq V_{2}$.

\section{Estimation Results}

This section presents a comparison between reactive power limits obtained from detailed and interpolation based equations. For this, the reactive power capacity of each machine is calculated at different $P$ and $V$ values.

The generic capability curves were built for $P_{\text {min }}=0.25$, $P_{\max }=0.85, V_{1}=0.99$ and $V_{2}=1.01$, all in pu on the machine base. These voltage values were arbitrary chosen considering that the machine will normally operate within this voltage range. Any $V$ value outside this interval can be evaluated as well.

Fig. 7 presents the synchronous machine reactive power limits estimated at $P=0.25,0.45,0.65$ and $0.85 \mathrm{pu}$ for $V=1.00 \mathrm{pu}$. The actual capability curve is also shown for comparison purposes.

The estimations shown in Fig. 7 were obtained using different $V_{1}$ and $V_{2}$. The first case uses [0.97 1.03] pu and the second one uses [0.99 1.01] pu. The latter gives slightly better estimations due to the closeness to $V=1.00 \mathrm{pu}$.

Note that at lagging power factor the estimation is less accurate at $P=0.45$ and $0.65 \mathrm{pu}$ due to the curvature of the actual capability curve. For leading power factor the

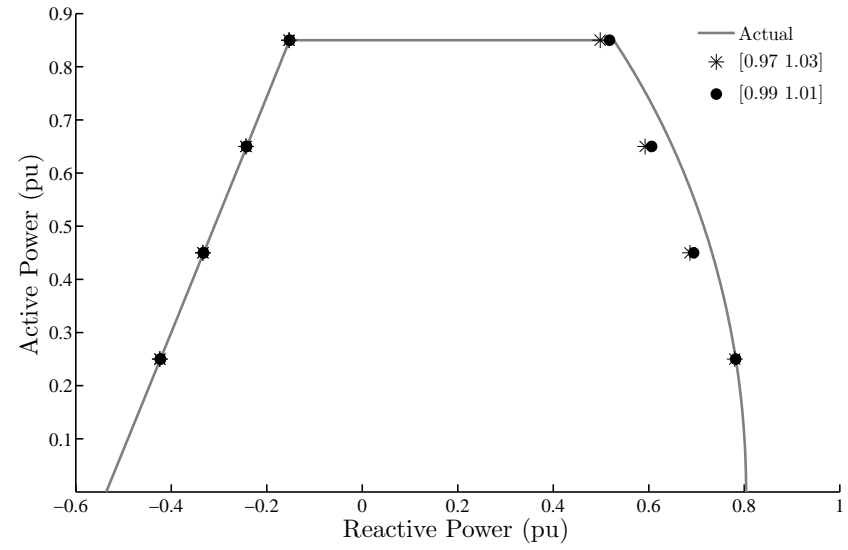

Figure 7. Actual versus estimated reactive power limits for $V=1 \mathrm{pu}$

Table I

ESTIMATION OF SYNCHRONOUS MACHINE REACTIVE POWER LIMITS

\begin{tabular}{cccccc}
\hline$P$ & $V$ & $Q_{\max }$ & $\%$ Error & $Q_{\min }$ & $\%$ Error \\
\hline 0.45 & 0.96 & 0.6858 & 6.64 & -0.2811 & 0.60 \\
0.65 & 0.96 & 0.5813 & 10.17 & -0.1903 & 0.90 \\
\hline 0.45 & 1.00 & 0.6939 & 5.25 & -0.3326 & 0.14 \\
0.65 & 1.00 & 0.6059 & 6.76 & -0.2425 & 0.19 \\
\hline 0.45 & 1.04 & 0.7021 & 2.87 & -0.3841 & 0.47 \\
0.65 & 1.04 & 0.6304 & 2.21 & -0.2946 & 0.62 \\
\hline
\end{tabular}

approximation (of $Q_{\text {min }}$ ) is very accurate because the subexcitation limitation is a straight line as the generic model.

Table I presents the estimated synchronous machine reactive power limits in pu and their percentage error with respect to the actual limits. The limits were estimated using $V_{1}=0.99 \mathrm{pu}$ and $V_{2}=1.01 \mathrm{pu}$. The comparisons show estimation results for low terminal voltage $\left(V<V_{1}\right)$, nominal voltage and high terminal voltage $\left(V>V_{2}\right)$. Due to the curvature of the actual capability curve, the largest percentage error is $10 \%$ for $P=$ $0.45 \mathrm{pu}$. The approximation is acceptable considering that the method is very simple. In addition, the estimated limits are more restrictive than the actual limits which gives a higher degree of security.

Tables II and III present the estimated reactive power limits of the DFIG and FCG, respectively. The estimations were carried out using the same $V_{1}$ and $V_{2}$ for the same $P$ and $V$ values. These percentage errors also demonstrate the proposed generic curves provide good approximations of the actual machine limits. Note that there is no guarantee that estimations are better if $V$ is within $V_{1}$ and $V_{2}$.

In general, the smallest percentage errors are obtained for those cases where the curvature of the actual capability curve is less pronounced. For example, the estimated $Q_{\max }$ in Table I is more accurate for $V=1.04 \mathrm{pu}$; here the curvature is less pronounced as shown in Fig. 6. Similarly, the estimations of $Q_{\max }$ in Table III are more accurate than $Q_{\min }$ 
Table II

ESTIMATION OF DFIG REACTIVE POWER LIMITS

\begin{tabular}{cccccc}
\hline$P$ & $V$ & $Q_{\max }$ & $\%$ Error & $Q_{\min }$ & $\%$ Error \\
\hline 0.45 & 0.96 & 0.6937 & 7.83 & -1.0813 & 4.56 \\
0.65 & 0.96 & 0.5772 & 9.88 & -0.9763 & 5.26 \\
\hline 0.45 & 1.00 & 0.7236 & 7.11 & -1.1152 & 4.30 \\
0.65 & 1.00 & 0.6138 & 8.76 & -1.0135 & 4.91 \\
\hline 0.45 & 1.04 & 0.7534 & 6.28 & -1.1492 & 4.00 \\
0.65 & 1.04 & 0.6503 & 7.48 & -1.0507 & 4.51 \\
\hline
\end{tabular}

Table III

ESTIMATION OF FCG REACTIVE POWER LIMITS

\begin{tabular}{cccccc}
\hline$P$ & $V$ & $Q_{\max }$ & $\%$ Error & $Q_{\min }$ & $\%$ Error \\
\hline 0.45 & 0.96 & 0.4448 & 1.45 & -0.9753 & 5.22 \\
0.65 & 0.96 & 0.4134 & 1.58 & -0.8555 & 6.60 \\
\hline 0.45 & 1.00 & 0.3277 & 3.38 & -1.0299 & 4.63 \\
0.65 & 1.00 & 0.2975 & 3.74 & -0.9169 & 5.74 \\
\hline 0.45 & 1.04 & 0.2106 & 2.60 & -1.0846 & 4.06 \\
0.65 & 1.04 & 0.1817 & 3.03 & -0.9783 & 4.89 \\
\hline
\end{tabular}

estimations because the curvature of the voltage limitation is less pronounced than the current limitation, see Fig. 4.

Other limiting factors not mentioned in this paper could be also included to obtain new points of the generic curves. Clearly, this will not affect the formulation to calculate the reactive power limits.

\section{CONClusion}

The use of fixed limits unnecessary restricts DG participation on voltage control and network optimization. This paper presented a simple formulation to calculate the reactive power limits of distributed generators as function of actual machine terminal voltage and active power production. The limits are obtained from generic capability curves as approximations of the actual curves. It was shown that the estimated limits are always more restrictive than the actual machine limits, providing a security margin. In addition, the comparisons showed that approximations are acceptable. The proposed approximations can be used for efficient estimation of generator reactive capabilities in on-line applications.

\section{ACKNOWLEDGEMENTS}

The authors would like to thank Dr. Thierry Van Cutsem, affiliated to The University of Liège and FNRS, for his helpful recommendations on the generic capability curve. They are also grateful to Mr. Gerardo Araya, graduate student at UCR, for his support in obtaining the DFIG capability curve.

\section{REFERENCES}

[1] K. Turitsyn, P. Sulc, S. Backhaus, and M. Chertkov, "Options for control of reactive power by distributed photovoltaic generators," Proc. IEEE, vol. 99, no. 6, pp. 1063-1073, Jun. 2011.
[2] M. Baran and I. El-Markabi, "A multiagent-based dispatching scheme for distributed generators for voltage support on distribution feeders," IEEE Trans. Power Syst., vol. 22, no. 1, pp. 52-59, Feb. 2007.

[3] L. Yu, D. Czarkowski, and F. de Leon, "Optimal distributed voltage regulation for secondary networks with dgs," IEEE Trans. Smart Grid, vol. 3, no. 2, pp. 959-967, Jun. 2012.

[4] P. Pachanapan, O. Anaya-Lara, A. Dysko, and K. Lo, "Adaptive zone identification for voltage level control in distribution networks with dg," IEEE Trans. Smart Grid, vol. 3, no. 4, pp. 1594-1602, Dec. 2012.

[5] G. Valverde and T. Van Cutsem, "Model predictive control of voltages in active distribution networks," IEEE Trans. Smart Grid, vol. 4, no. 4, pp. 2152-2161, Dec. 2013.

[6] T. Van Cutsem and C. Vournas, Voltage Stability of Electric Power Systems. Springer, 1998.

[7] C. W. Taylor, Power System Voltage Stability. Mc Graw Hill, 1994

[8] M. Adibi and D. P. Milanicz, "Reactive capability limitation of synchronous machines," IEEE Trans. Power Syst., vol. 9, no. 1, pp. 29-40, Feb. 1994.

[9] M. S. Sarma, Electric Machines, 2nd ed. Brooks/Cole, 1994.

[10] F. Van Meulebroeke, "Modelling of voltage collapse including dynamic phenomena," CIGRÉ Task Force, Tech. Rep., 1993.

[11] T. Lund, P. Sorensen, and J. Eek, "Reactive power capability of a wind turbine with doubly fed induction generator," Wind Energy, vol. 10, no. 4, pp. 379-394, Jul. 2007.

[12] S. Engelhardt, I. Erlich, C. Feltes, J. Kretschmann, and F. Shewarega, "Reactive power capability of wind turbines based on doubly fed induction generators," IEEE Trans. Energy Conver., vol. 26, no. 1, pp. 364-372, Mar. 2011.

[13] B. Singh and S. N. Singh, "Reactive capability limitations of doubly-fed induction generators," Electric Power Components and Systems, vol. 37, no. 4, pp. 427-440, Apr. 2009.

[14] M. Kayikci and J. Milanovic, "Reactive power control strategies for dfig-based plants," IEEE Trans. Energy Convers., vol. 22, no. 2, pp. 389-396, Jun. 2007.

[15] N. Ullah, K. Bhattacharya, and T. Thiringer, "Wind farms as reactive power ancillary service providers - technical and economic issues," IEEE Trans. on Energy Convers., vol. 24, no. 3, pp. 661-672, Sep. 2009.

[16] M. Chinchilla, S. Arnalte, J. Burgos, and J. Rodríguez, "Power limits of grid-connected modern wind energy systems," Renewable Energy, vol. 31, no. 9, pp. 1455 - 1470, Jul. 2006.

[17] V. Calderaro, G. Conio, V. Galdi, G. Massa, and A. Piccolo, "Optimal decentralized voltage control for distribution systems with inverter-based distributed generators," IEEE Trans. Power Syst., vol. 29, no. 1, pp. 230241, Jan. 2014

\section{APPENDIX}

The exact capability curves were calculated using the following machine parameters.

Table IV

SYNCHRONOUS MACHINE PARAMETERS

\begin{tabular}{lccccc}
\hline$S_{\text {nom }}$ & $3.25 \mathrm{MVA}$ & $X_{l}$ & $0.124 \mathrm{pu}$ & $m$ & $0.10 \mathrm{pu}$ \\
$V_{\text {nom }}$ & $4.16 \mathrm{kV}$ & $X_{d}$ & $2.013 \mathrm{pu}$ & $n$ & $6.03 \mathrm{pu}$ \\
$I_{a}^{\text {max }}$ & $1.00 \mathrm{pu}$ & $X_{q}$ & $1.980 \mathrm{pu}$ & $I_{f}^{\max }$ & $2.81 \mathrm{pu}$ \\
\hline
\end{tabular}

Table V

DFIG PARAMETERS

\begin{tabular}{cccccc}
\hline$P_{\text {nom }}$ & $1.5 \mathrm{MW}$ & $R_{r}$ & $0.005 \mathrm{pu}$ & $I_{s}^{\max }$ & $1.00 \mathrm{pu}$ \\
$V_{\text {nom }}$ & $575 \mathrm{~V}$ & $X_{l s}$ & $0.171 \mathrm{pu}$ & $I_{r}^{\max }$ & $1.00 \mathrm{pu}$ \\
Poles & 6 & $X_{l r}$ & $0.156 \mathrm{pu}$ & $V_{r}^{\max }$ & $0.30 \mathrm{pu}$ \\
$R_{s}$ & $0.007 \mathrm{pu}$ & $X_{m}$ & $2.900 \mathrm{pu}$ & $I_{G S C}^{\max }$ & $0.25 \mathrm{pu}$ \\
\hline
\end{tabular}

Table VI

FCG PARAMETERS

\begin{tabular}{lccccc}
\hline$P_{\text {nom }}$ & $1.5 \mathrm{MW}$ & $X_{c}$ & $0.30 \mathrm{pu}$ & $I_{c}^{\max }$ & $1.17 \mathrm{pu}$ \\
$V_{\text {nom }}$ & $380 \mathrm{~V}$ & - & - & $V_{c}^{\max }$ & $1.11 \mathrm{pu}$ \\
\hline
\end{tabular}

